\title{
Pre-hCG variables associated with occurrence of ascites in IVF/ICSI patients at moderate risk of developing OHSS: A pilot investigation
}

\author{
Manuel Fernández-Sánchez ${ }^{*}$, Per Broberg ${ }^{2}$, Göran Pettersson $^{3}$, Cristiano Busso $^{4}$, Antonio Pellicer $^{1}$, \\ Joan-Carles Arce ${ }^{3}$ \\ ${ }^{1}$ Instituto Valenciano de Infertilidad (IVI) Sevilla, Sevilla, Spain \\ ${ }^{2}$ Biometrics, Global Clinical \& Non-Clinical R\&D, Ferring Pharmaceuticals A/S, Copenhagen, Denmark \\ ${ }^{3}$ Reproductive Health, Global Clinical \& Non-Clinical R\&D, Ferring Pharmaceuticals A/S, Copenhagen, Denmark \\ ${ }^{4}$ International Vaccine Institute (IVI) Valencia, Valencia, Spain \\ Email: "
}

Received 19 October 2012; revised 21 November 2012; accepted 30 November 2012

\begin{abstract}
Objective: To identify predictors of ascites collected prior to the hCG administration in patients undergoing IVF/ICSI treatment at moderate risk of developing moderate/severe ovarian hyperstimulation syndrome (OHSS), and, based on these predictors, develop a nomogram for estimation of the probability of presence of ascites. Methods and Materials: Data were derived from 53 patients with 20 - 30 follicles $\geq 10 \mathrm{~mm}$ at end of stimulation. All patients received a single dose of hCG $(250 \mathrm{mg})$ to trigger final follicular maturation when $\geq 2$ follicles of $\geq 18 \mathrm{~mm}$ were observed. Transvaginal ultrasound to measure ascites (total amount of peritoneal fluid $\geq 9 \mathrm{~cm}^{2}$ in lithotomy position) was performed 2,5 and 8 days after the hCG administration. Associations between clinical, sonographic and endocrinological variables recorded prior to the hCG administration and presence of ascites were analyzed by univariable and multivariable logistic regression. Results: Thirty-four patients (64\%) had ultrasonic evidence of ascites. The multivariable analysis identified the total number of follicles [OR $1.29(95 \%$ CI: $1.02-1.69, P=0.043)]$, the ovarian volume [OR $1.05(95 \%$ CI: $1.00-1.11, P=0.047)]$ and BMI [OR $0.76(95 \%$ CI: $0.56-0.99, P=0.053)]$ as predictors of ascites (AUC $=\mathbf{0 . 8 2 5}$ ). A nomogram (PROFET) was designed with these three variables for individual prediction of the probability of development of ascites. Conclusions: This pilot investigation indicates that the risk of peritoneal fluid accumulation in IVF/ICSI patients at moderate risk of developing moderate/severe OHSS is influenced by the number of follicles and the ovarian volume on the day of hCG administration as well as the BMI.
\end{abstract}

\footnotetext{
${ }^{*}$ Corresponding author.
}

Keywords: Ascites; IVF; OHSS; Ovarian Stimulation; Prediction

\section{INTRODUCTION}

The diagnosis of ovarian hyperstimulation syndrome (OHSS) is based on a spectrum of signs and symptoms, and different classification systems to grade the severity have been suggested; for review, see Golan and Weissman [1]. The earliest classification schemes were based on clinical presentation and laboratory findings [2,3]. During the 1980s, transvaginal ultrasonography (TVU) became available and a revised classification of OHSS was introduced that included ultrasound-based detection of accumulation of fluid in the peritoneal cavity (ascites) [4]. This classification scheme is still widely employed although modifications for the classification of the most severe forms have been proposed $[1,5,6]$.

Effective prevention of OHSS is obviously better than current empirical treatment [7-9] and recognition of potential risk factors prior to start of ovarian stimulation is key to preventing the syndrome; for review, see Delvigne [10] and Papanikolaou et al. [11]. Primary risk factors suggested in the literature include polycystic ovarian syndrome, young age, low body weight, a high antral follicle count, a high serum concentration of anti-Müllerian hormone, use of gonadotropin releasing hormone $(\mathrm{GnRH})$ analogues in a long protocol, higher doses of exogenous gonadotropins, and human chorionic gonadotropin (hCG) to trigger final follicular maturation [9,12-19].

Both in patients with and without any known predisposing factor of OHSS, a number of potential secondary risk factors occurring during ovarian stimulation have been suggested such as a high absolute and/or rapidly rising level of serum estradiol, a high number of preovulatory follicles and a high number of oocytes re- 
trieved [12,13,20-22]. There is, however, a considerable overlap of the distribution of values for each ovarian response variable between patients who develop OHSS and those who demonstrate a normal response, and none of these variables are considered to be independently predictive of OHSS [10,11]. Efforts have therefore been made to improve the prognostic power by combining secondary risk factors. Several investigators have used serum estradiol levels together with follicle number and/or number of oocytes retrieved to predict the occurrence of OHSS, although with somewhat limited success [23-25].

Prevention or reduction of the incidence and severity of OHSS in patients who have progressed to high risk during the treatment cycle implies the possibility to delay or withhold the administration of hCG. Assessment of variables that can identify these patients before triggering of final follicular maturation may therefore help clinicians to undertake secondary preventive methods including coasting, reduction of the hCG dose or use of GnRH agonist for triggering of final follicular maturation, use of dopamine agonist, freezing of all embryos, or withholding of hCG and cycle cancellation [8,9,26-29].

The main objective of this pilot investigation was to develop a multivariable model for prediction of ultrasonic evidence of ascites in individual cases prior to the hCG administration using a cohort of patients undergoing controlled ovarian stimulation (COS) for assisted reproduction technologies (ART). All patients were presumed to be at moderate risk of developing moderate/ severe OHSS based on the presence of 20 - 30 preovulatory follicles. Ultrasonic evidence of ascites was chosen as the dependent variable in the logistic regression analyses, since it is a quantitative measure of moderate/ severe OHSS unlike more subjective variables such as abdominal discomfort, distension and pain.

\section{METHODS AND MATERIALS}

This is a retrospective investigation of 53 in vitro fertilization (IVF)/intracytoplasmic sperm injection (ICSI) patients who were included in the placebo arm of a double-blind, randomized controlled trial comparing quinagolide versus placebo in prevention of OHSS (ClinicalTrials.gov Identifier: NCT00329693) [30]. The trial was conducted in accordance with the Declaration of Helsinki and Good Clinical Practice. The protocol, the subject information sheet and the consent form were reviewed and approved by the independent ethics committees and the regulatory authorities prior to trial initiation. All participants gave written, signed, informed consent prior to entering the trial.

The patients were 21 - 37 years old, had a BMI of 18 $29 \mathrm{~kg} / \mathrm{m}^{2}$ and early follicular phase serum concentrations of FSH within normal limits $(1-12 \mathrm{IU} / \mathrm{l})$ as well as a uterus consistent with expected normal function and presence of both ovaries. They had been infertile for at least 1 year and were undergoing a COS cycle for IVF/ICSI at the time of the trial in which they presented with ovarian response suggestive of being at moderate risk of developing moderate/severe OHSS because of the presence of at least 20 follicles of $10 \mathrm{~mm}$ or greater on the day of hCG administration. Patients considered to be at high risk of developing OHSS, i.e. presenting with more than 30 follicles and/or serum estradiol of $\geq 6000 \mathrm{pg} / \mathrm{ml}$ at the time of hCG administration, were not included as clinical practice in the participating centers for these cases was either cycle cancellation or most likely of GnRH agonist administration to trigger final follicle maturation. Furthermore, patients were not included if coasting or other preventive measures for managing OHSS had been applied.

All patients received a single dose of hCG (Ovitrelle $250 \mathrm{mg}$ s.c., Merck-Serono, Geneva, Switzerland) to trigger final follicular maturation when $\geq 2$ follicles of $\geq 18 \mathrm{~mm}$ were observed. Prior to the administration of hCG, measurement of serum estradiol, physical and gynecological examination, body measurements (body weight, height and waist circumference), and TVU of ovaries (size of ovaries, number of follicles according to size) and uterus (endometrial thickness) were performed. Oocyte retrieval took place $36 \pm 2$ hours after the hCG administration and transfer of one or two embryos was done on day 3, 5 or 6 after the oocyte retrieval. Luteal support was provided with vaginal progesterone (Utrogestan, SEID Laboratories, Barcelona, Spain), $200 \mathrm{mg}$ twice daily from the day after oocyte retrieval to negative $\beta h C G$ test or to the day of clinical pregnancy assessment.

TVU was employed to measure the peritoneal fluid pockets in the pelvis on day 2 (just prior to the oocyte retrieval) as well as 5 and 8 days after the hCG administration. The size of each pocket was determined by measuring the greatest diameter and its greatest perpendicular diameter and multiplying these two numbers giving the unit $\mathrm{cm}^{2}$. In line with the definition used in a previous study in which the value of a dopamine agonist to prevent OHSS after COS was examined [26], the existence of ultrasonic evidence of ascites was confirmed when the total size of pockets of peritoneal fluid $\geq 9 \mathrm{~cm}^{2}$ was observed when the patient was in lithotomy position (i.e. with the gynecological table at $45^{\circ}$ from the floor of the room). This cut-off was based on the mean +2 standard deviations of the value $\left(3.5 \pm 2.8 \mathrm{~cm}^{2}\right)$ found after oocyte retrieval in women who showed no risk of OHSS. Furthermore, significantly more placebo-treated women had peritoneal fluid $\geq 9 \mathrm{~cm}^{2}$ than patients treated with dopamine agonist in the study by Álvarez et al. [26]. 
These observations indicate that it is clinically relevant to use a cut-off of $9 \mathrm{~cm}^{2}$ in prognostic models of moderate/severe OHSS.

\section{Data Analysis}

All statistical analyses were performed in " $R$ ", version 2.7.0 (R Development Core Team, 2008). The variables assessed prior to the $\mathrm{hCG}$ administration were included in a univariable logistic regression analysis as the first step to identify variables associated with ultrasonic evidence of ascites. Treatment differences are presented with odds ratios (OR) and two-sided $95 \%$ confidence interval (CI) and corresponding $\mathrm{P}$ values. The Higher Criticism test [31] was used to select variables that would qualify to be entered in the multivariable regression analysis. This method yielded a cut-off for the $\mathrm{P}$ values from the univariable logistic regression, such that variables with $\mathrm{P}$ values equal to or less than the threshold were included in the multivariable modeling. The multivariable logistic regression model emerged from a backward and forward stepwise procedure that sought to optimize model fit with respect to the Akaike information criterion. The predictive ability of the model was assessed by determining the area under the receive roperating characteristics (ROC) curve.

\section{RESULTS}

Of the 53 patients included in this investigation, 34 (64\%) had ultrasonic evidence of ascites within 9 days after the hCG administration. Table 1 shows the baseline characteristics and variables recorded in the treatment cycle prior to the hCG administration in the subgroups of patients with and without ultrasonic evidence of ascites.

The univariable logistic regression analysis of the variables assessed prior to the $\mathrm{hCG}$ administration yielded a P value cut-off of 0.321 in the Higher Criticism test and the following variables were included in the multivariable logistic regression model search: 1) Demographics: body weight, BMI, waist circumference; 2) Infertility history: number of IVF cycles, number of ICSI cycles, total number of IVF/ICSI cycles; 3) Menstrual history: menstrual cycle length; 4) Obstetric history: method of conception: ICSI; and 5) Day of hCG: ovarian volume, total number of follicles, number of follicles $\geq$ $15 \mathrm{~mm}$, number of follicles $\geq 10 \mathrm{~mm}$, number of follicles $<10 \mathrm{~mm}$, and serum estradiol. Female age was also added to the multivariable analysis, since this variable has been found to be associated with OHSS in other studies.

Using backward and forward stepwise selection, the best model for prediction of the probability of ultrasonic evidence of ascites was obtained using the variables total number of follicles [OR 1.29 (95\% CI: $1.02-1.69), \mathrm{P}=$
0.043], ovarian volume [OR 1.05 (95\% CI: $1.00-1.11)$, $\mathrm{P}=0.047$ ] and BMI [OR 0.76 (95\% CI: $0.56-0.99), \mathrm{P}=$ 0.053] (Table 2). The predictive ability of the model measured by the area under the ROC curve (AUC) was 0.825 (Figure 1). The AUC values of total number of follicles, ovarian volume and BMI obtained in the univariate logistic regression analysis for prediction of ascites were $0.673,0.776$ and 0.638 , respectively, which showed that the power of prediction increased when the multivariate model that included all three variables was used.

Figure 2 illustrates the relationships between the total number of follicles, ovarian volume and BMI for patients with ultrasonic evidence of ascites and those with absence of ascites. As can be seen, the estimated probability of ultrasonic evidence of ascites is increased by a high number of follicles and a large ovarian volume on the day of hCG, as well as a low BMI. The multivariable logistic regression model was then used to design a nomogram (PROFET) for prediction of the likelihood of developing ascites following gonadotrophin stimulation and hCG triggering of final follicle maturation for a given IVF/ICSI patient (Figure 3). The PROFET nomogram includes three steps. By drawing vertical lines from the values related to each of the three variables to the scale at the top of the nomogram a number of points will be obtained for each variable. The total score is then summed up and the probability of ultrasonic evidence of ascites can subsequently be estimated by drawing a vertical line from the value on the "total score scale" to the "probability scale" at the bottom of the nomogram.

\section{DISCUSSION}

This pilot investigation included a cohort of patients who underwent a COS cycle for IVF/ICSI treatment in whom an ovarian response suggestive of being at risk of developing OHSS was observed. Moderate OHSS is usually defined as the presence of mild symptoms (abdominal distension and discomfort, and enlarged ovaries) plus the appearance of peritoneal fluid [4]. As low or moderate amounts of peritoneal fluid can be quantitatively measured by TVU at an early stage, even in the absence of clinical evidence of third space fluid accumulation, ultrasonic evidence of ascites was chosen as the dependent variable in the prediction model of moderate/severe OHSS.

The multivariable logistic regression analysis in the present investigation showed that three variables, i.e. number of follicles, ovarian volume and BMI, were predictive of peritoneal fluid accumulation and that the power of prediction increased when a model that includes several variables was used. Concerning BMI, afew studies have investigated the impact of this variable 
Table 1. Baseline characteristics and variables obtained at the end of gonadotrophin stimulation of patients with absence $\left(<9 \mathrm{~cm}^{2}\right)$ or presence $\left(\geq 9 \mathrm{~cm}^{2}\right)$ of ultrasonic evidence of ascites.

\begin{tabular}{|c|c|c|c|c|c|c|}
\hline & & Absence $(n=19)$ & Presence $(n=34)$ & OR & $95 \% \mathrm{CI}$ & P value ${ }^{c}$ \\
\hline \multirow[t]{4}{*}{ Demographics } & Female age (years) & $30.6 \pm 2.8$ & $31.1 \pm 2.6$ & 1.08 & $0.87-1.34$ & 0.500 \\
\hline & Height (m) & $1.63 \pm 0.06$ & $1.63 \pm 0.06$ & $1.18^{\mathrm{a}}$ & $0.46-3.16$ & 0.727 \\
\hline & Body weight (kg) & $62.4 \pm 8.2$ & $59.6 \pm 7.4$ & 0.95 & $0.88-1.03$ & 0.205 \\
\hline & BMI $\left(\mathrm{kg} / \mathrm{m}^{2}\right)$ & $23.6 \pm 2.5$ & $22.4 \pm 2.6$ & 0.84 & $0.66-1.04$ & 0.121 \\
\hline Menstrual history & Menstrual cycle length (days) & $30.4 \pm 8.5$ & $35.5 \pm 18.8$ & 1.03 & $0.99-1.11$ & 0.298 \\
\hline \multirow[t]{7}{*}{ Infertility history } & Duration of infertility (years) & $2.51 \pm 1.44$ & $2.41 \pm 2.70$ & 0.98 & $0.76-1.31$ & 0.884 \\
\hline & Primary reason of infertility & & & & & \\
\hline & Anovulatory infertility, n (\%) & $2(11)$ & $4(12)$ & 1.13 & $0.20-8.79$ & 0.892 \\
\hline & Endometriosis, n (\%) & $0(0)$ & $1(3)$ & - & - & 0.997 \\
\hline & Male factor, n (\%) & $12(63)$ & $19(56)$ & 0.79 & $0.10-4.74$ & 0.792 \\
\hline & Unexplained infertility, n (\%) & $4(21)$ & $4(12)$ & 0.50 & $0.05-4.33$ & 0.535 \\
\hline & Other, n (\%) & $0(0)$ & $2(6)$ & - & - & 0.995 \\
\hline \multirow[t]{14}{*}{ Cycle history } & Ovulation induction cycles (n) & & & & & \\
\hline & Clomiphene citrate & $0.32 \pm 1.38$ & $0.21 \pm 0.73$ & 0.90 & $0.48-1.69$ & 0.701 \\
\hline & Gonadotrophin & $1.05 \pm 1.72$ & $1.03 \pm 1.64$ & 0.99 & $0.71-1.42$ & 0.960 \\
\hline & Other & $0.42 \pm 1.30$ & $0.18 \pm 1.03$ & 0.83 & $0.46-1.39$ & 0.461 \\
\hline & Total & $1.79 \pm 2.37$ & $1.41 \pm 1.97$ & 0.92 & $0.70-1.20$ & 0.529 \\
\hline & IVF/ICSI cycles (n) & & & & & \\
\hline & ICSI & $0.42 \pm 0.84$ & $0.18 \pm 0.52$ & 0.57 & $0.22-1.34$ & 0.209 \\
\hline & Total & $0.74 \pm 1.10$ & $0.27 \pm 0.71$ & 0.55 & $0.27-1.04$ & 0.076 \\
\hline & Method of conception (yes/no) & & & & & \\
\hline & IVF & $1 / 18$ & $0 / 34$ & 0.00 & - & 0.991 \\
\hline & ICSI & $2 / 17$ & $1 / 33$ & 0.26 & $0.01-2.87$ & 0.282 \\
\hline & Pregnancies (n) & $0.32 \pm 0.67$ & $0.24 \pm 0.65$ & 0.83 & $0.35-2.05$ & 0.667 \\
\hline & Ectopic pregnancies (yes/no) & $1 / 18$ & $0 / 34$ & 0.00 & - & 0.991 \\
\hline & Miscarriages (yes/no) & $2 / 17$ & $3 / 31$ & 0.82 & $0.12-6.71$ & 0.839 \\
\hline \multirow[t]{9}{*}{ Current cycle (day of hCG) } & Serum estradiol (pg/ml) & $2753 \pm 1297$ & $3100 \pm 1179$ & $1.01^{\mathrm{b}}$ & $0.99-1.04$ & 0.321 \\
\hline & Ovarian volume (ml) & $45.5 \pm 18.6$ & $59.0 \pm 15.6$ & 1.06 & $1.02-1.11$ & 0.012 \\
\hline & Endometrial thickness (mm) & $10.6 \pm 1.38$ & $11.1 \pm 2.17$ & 1.15 & $0.85-1.60$ & 0.378 \\
\hline & Follicles (n): & & & & & \\
\hline & $\geq 15 \mathrm{~mm}$ & $13.8 \pm 3.3$ & $15.2 \pm 4.3$ & 1.10 & $0.95-1.28$ & 0.220 \\
\hline & $<15 \mathrm{~mm}$ & $9.2 \pm 3.6$ & $10.0 \pm 4.6$ & 1.05 & $0.92-1.21$ & 0.500 \\
\hline & $\geq 10 \mathrm{~mm}$ & $22.6 \pm 2.01$ & $24.2 \pm 3.60$ & 1.19 & $0.99-1.47$ & 0.085 \\
\hline & $<10 \mathrm{~mm}$ & $0.37 \pm 0.83$ & $0.97 \pm 1.57$ & 1.55 & $0.94-3.25$ & 0.148 \\
\hline & Total & $23.0 \pm 2.1$ & $25.2 \pm 3.6$ & 1.27 & $1.05-1.60$ & 0.025 \\
\hline
\end{tabular}

${ }^{\mathrm{a}}$ Steps of $10 \mathrm{~cm}$; ${ }^{\mathrm{b}}$ Steps of $50 \mathrm{pg} / \mathrm{ml} ;{ }^{\mathrm{c}}$ Univariable logistic regression. 
Table 2. Multivariable logistic regression analysis of variables obtained prior to the hCG administration for the prediction of ultrasonic evidence of ascites $\left(\geq 9 \mathrm{~cm}^{2}\right)$ in IVF/ICSI cycles.

\begin{tabular}{ccccc}
\hline Predictors & Estimate & OR & $95 \%$ CI & P value \\
\hline (Intercept) & -1.861 & - & - & - \\
Number of follicles & 0.254 & 1.29 & $1.02-1.69$ & 0.043 \\
Ovarian volume & 0.050 & 1.05 & $1.00-1.11$ & 0.047 \\
BMI & -0.275 & 0.76 & $0.56-0.99$ & 0.053 \\
\hline
\end{tabular}

OR (odds ratio $)=\exp (-1.861+0.254$ [number of follicles] +0.050 [ovarian volume $]-0.275[\mathrm{BMI}]) ; \mathrm{P}$ (probability of ascites) $=\mathrm{OR} /(\mathrm{OR}+1) . \mathrm{CI}=$ confidence interval.

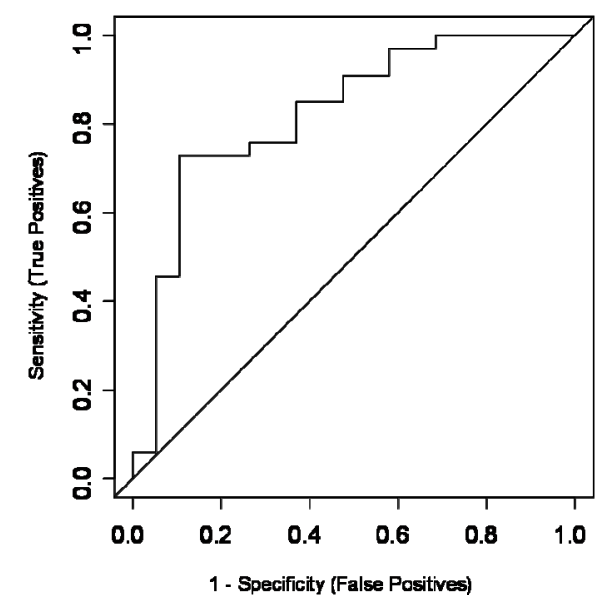

Figure 1. ROC plot displaying sensitivity and specificity of the predictive model of ultrasonic evidence of ascites $\left(\geq 9 \mathrm{~cm}^{2}\right)$. The AUC of the model $=0.825(95 \% \mathrm{CI}: \pm 0.12)$.

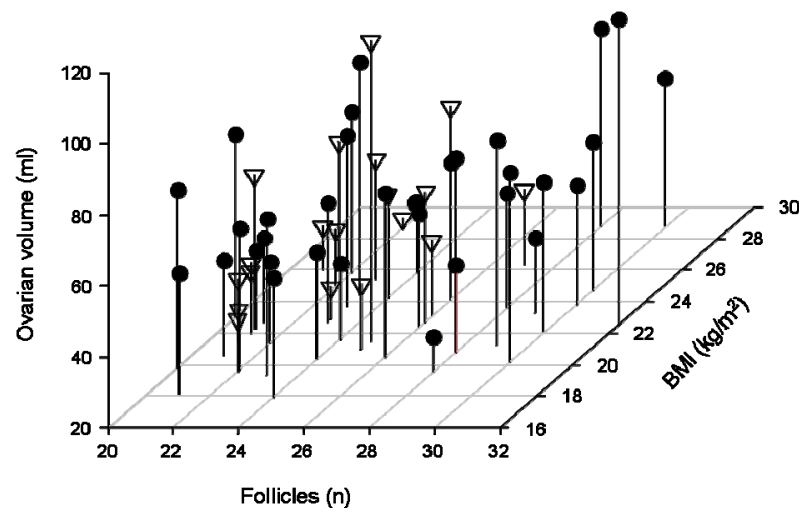

Figure 2. Relationships between number of follicles (x-axis) and ovarian volume (y-axis) on the day of hCG and BMI (z-axis) for patients with absence $\left(<9 \mathrm{~cm}^{2}\right.$, triangles) or presence $\left(\geq 9 \mathrm{~cm}^{2}\right.$, circles) of ultrasonic evidence of ascites.

(or body weight) on the risk of developing OHSS and there are conflicting reports in the literature. Navot et al. [5], Aramwit et al. [25] and Danninger et al. [32] observed that patients with OHSS had lower body weight/ BMI than non-OHSS patients, while other investtiga- tors were not able to demonstrate any difference $[12,13]$. In the present investigation, which included women with a BMI of less than $30 \mathrm{~kg} / \mathrm{m}^{2}$, there was a tendency $(\mathrm{P}=$ 0.121 ) in the univariable analysis towards lower BMI in the group of patients with ultrasonic evidence of ascites. In the multivariable logistic regression model, however, BMI was found to be borderline significant and improved the model fit with respect to the Akaike information criterion. BMI was therefore included in the final model predicting presence of ascites [OR $0.76(95 \% \mathrm{CI}$ : $0.56-0.99, \mathrm{P}=0.053)]$ implying that lean patients may be at higher risk of having fluid accumulation in the pelvis.

In contrast to earlier reports where patients suffering from OHSS have been reported to be significantly younger than those who did not develop the syndrome $[12,13,25,33]$, the mean ages of the patients in the present investigation were similar in the subgroup in whom ascites developed and in the subgroup of patients in whom there was no ultrasonic evidence of ascites. There are no obvious explanation for the lack of an inverse correlation between age and existence of ascites in our investigation except that most of the patients were relatively young and of similar age [mean: $30.9 \pm 2.7$ years (95\% CI: $30.2 ; 31.6)]$. Nevertheless, the reported differences in mean age in the literature are small and age is not considered to be a suitable single predictor for OHSS [10].

It has been shown in several earlier studies that a high number of large pre-ovulatory follicles is a secondary risk factor for OHSS, although the classification of follicle size varies between studies $[5,13,20,21]$. On the other hand, if a patient develops less than 20 large follicles it is generally considered that the risk of developing severe OHSS is low $[23,34]$. In spite of the fact that the present investigation included patients at moderate risk because of the presence of $20-30$ follicles of $10 \mathrm{~mm}$ or greater on the day of hCG, the univariable analysis showed that including all follicles observed, independent of size, was significantly higher in the patients with ultrasonic evidence of ascites compared with the patients who had no evidence. There was a tendency as well towards more follicles of $10 \mathrm{~mm}$ or greater in the patients with ultrasonic evidence of ascites. Hence, the results of the present investigation suggest that it may be clinically relevant to consider not only the number of larger follicles but also the total number of follicles observed. It should be noted that patients considered to be at high risk, i.e. with $>30$ follicles $\geq 10 \mathrm{~mm}$ and/or serum estradiol $\geq 6000$ $\mathrm{pg} / \mathrm{ml}$ at end of COS, were not included in the prediction model as the objective was to predict the probability of occurrence of ascites in patients at moderate risk.

Ovarian volumes have been used earlier to classify patients who have already received hCG as having 


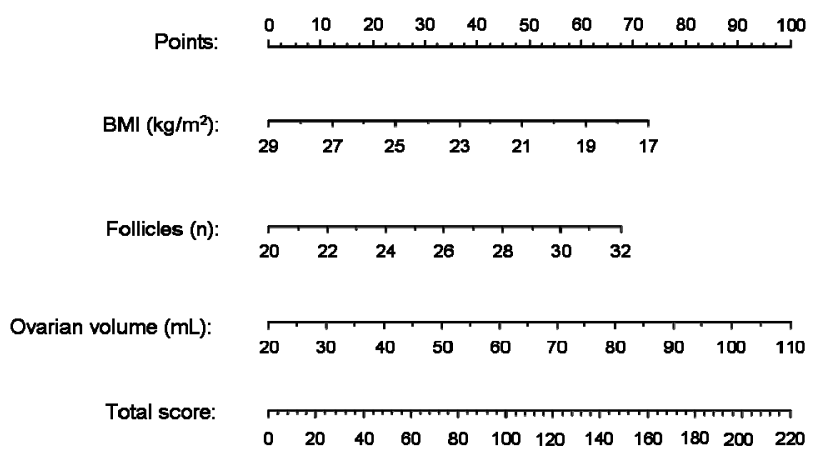

Probability of ascites:

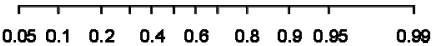

Figure 3. Profet nomogram for estimation of the probability of ultrasonic evidence of ascites prior to triggering of final follicle maturation in an IVF/ICSI cycle. The probability can be assessed using the number of follicles and the ovarian volume on the day of hCG, and BMI. The nomogram comprises three steps. By drawing vertical lines from the values related to each variable to the 'point scale' at the top of the nomogram, a number of points (a score) will be obtained for each variable. The total score is then calculated and the probability of ultrasonic evidence of ascites can be estimated by drawing a vertical line from the value on the "total score scale" to the "probability scale" at the bottom of the nomogram. For example, a patient presenting with a BMI of $28 \mathrm{~kg} / \mathrm{m}^{2}$, a total of 20 follicles and an ovarian volume of $40 \mathrm{ml}$ on the day of hCG will obtain $6+0$ $+22=28$ points in the nomogram. Her probability to develop ultrasonic evidence of ascites is estimated to be less than $10 \%$, while a patient with BMI, total number of follicles and ovarian volume of $22 \mathrm{~kg} / \mathrm{m}^{2}, 28$ and $60 \mathrm{ml}$, respectively, will obtain 43 $+45+44=132$ points and is estimated to have $90 \%$ probability.

different grades of OHSS [4,35]. In the present investigation, TVU of the ovaries was performed on the day of hCG prior to administration and the univariable analysis demonstrated that the mean ovarian volume was significantly higher in the patients with ultrasonic evidence of ascites within 9 days after the hCG administration compared with the patients who had no evidence of ascites. Furthermore, the multivariable logistic regression analysis showed that the ovarian volume was an independent predictor of ascites. This finding suggests that TVU measurement of ovarian volume may be included in the screening strategy for moderate/severe OHSS, which is in agreement with earlier observations in a study in patients undergoing IVF treatment [36]. This research group measured the total ovarian volume in patients considered to have an exaggerated response to the ovarian stimulation and they observed that the total ovarian volume prior to hCG administration was significantly higher in patients who progressed to moderate or severe OHSS compared with controls. Interestingly, a significantly higher mean ovarian volume has also been observed at stimulation day 1 in IVF patients who developed OHSS com- pared with those who demonstrated a normal response [32]. These authors suggested that early volumetry of the ovaries could help to detect patients at risk and prevent the occurrence of OHSS by early adjustment of the gonadotrophin dosage. In contrast, Jayaprakasan et al. [17], who performed TVU scans in the early follicular phase of the menstrual cycle preceding IVF treatment, were not able to demonstrate a significant difference in ovarian volume between the patients who developed moderate/ severe OHSS and those who had a normal ovarian response.

The serum estradiol concentration on the day of hCG was not a predictor of ascites in the present investigation, which is in marked contrast to several earlier studies. Many investigators have shown that an elevated concentration of estradiol during ovarian stimulation constitutes a risk factor for OHSS [3,5,22,23,35,37,38]. However, as mentioned above, patients presented with serum estradiol of $\geq 6000 \mathrm{pg} / \mathrm{ml}$ and/or more than 30 follicles, or where coasting or other preventive measures for managing OHSS had been applied, were not included in the present study cohort. This may explain why estradiol was not a predictor of ascites in our model. Moreover, considering a high estradiol level as the only predictive factor is not sufficiently accurate, since a large overlap in estradiol values has been found between patients who develop OHSS and those who do not $[5,12,39]$.

To increase the clinical usefulness of the findings in the multivariable model, a nomogram was developed for estimation of the probability of presence of ascites prior to triggering of final follicular maturation in an IVF/ICSI cycle. On the basis of the PROFET nomogram, the predicted probability may thus vary from very low to very high. For example, a patient with a BMI of 28 $\mathrm{kg} / \mathrm{m}^{2}$, a total of 20 follicles and an ovarian volume of 40 $\mathrm{ml}$ on the day of hCG is estimated to have a less than $10 \%$ probability of developing ascites, while a patient with BMI, number of follicles and ovarian volume of 22 $\mathrm{kg} / \mathrm{m}^{2}, 28$ and $60 \mathrm{ml}$, respectively, is estimated to have more than $90 \%$ probability. In the latter case, our model indicates that appropriate preventive strategies should be taken. In this context, it should be noted that prediction models often overestimate the predictive capacity when assessed in the same cohort in whom they have been developed $[40,41]$. Thus, an external validation of the present nomogram is needed to define its applicability in detecting patients at risk of developing moderate or severe OHSS. Until then, the model should be considered mainly indicative of risk rather than predictive.

In conclusion, the results of this investigation pointed out that higher number of follicles and larger ovarian volume on the day of hCG administration together with a low BMI may increase the risk of ultrasonic evidence of ascites in IVF/ICSI patients at risk of developing OHSS. 


\section{ACKNOWLEDGEMENTS}

The study was sponsored by Ferring Pharmaceuticals A/S.

\section{REFERENCES}

[1] Golan, A. and Weissman, A. (2009) Symposium: Update on prediction and management of OHSS. A modern classification of OHSS. Reproductive Biomedicine Online, 19, 28-32. doi:10.1016/S1472-6483(10)60042-9

[2] Rabau, E., David, A., Serr, D.M, Mashiach, S. and Lunenfeld, B. (1967) Human menopausal gonadotropins for anovulation and sterility. Results of 7 years of treatment. American Journal of Obstetrics and Gynecology, 98, 92-98.

[3] Schenker, J.G. and Weinstein. D. (1978) Ovarian hyperstimulation syndrome: A current survey. Fertility and Sterility, 30, 255-268.

[4] Golan, A., Ronel, R., Herman, A., Soffer, Y., Weinraub, Z. and Caspi, E. (1989) Ovarian hyperstimulation syndrome: An update review. Obstetrical and Gynecological Survey, 44, 430-440. doi:10.1097/00006254-198906000-00004

[5] Navot, D., Relou, A., Birkenfeld, A., Rabinowitz, R., Brzezinski, A. and Margalioth, E.J. (1988) Risk factors and prognostic variables in the ovarian hyperstimulation syndrome. American Journal of Obstetrics and Gynecology, 159, 210-215.

[6] Rizk, B. and Aboulghar, M.A. (1999) Classification, pathophysiology and management of ovarian hyperstimulation syndrome. In: Brinsden, P., Ed., In-Vitro Fertilization and Assisted Reproduction. The Parthenon Publishing Group, New York, London, 131-155.

[7] Delvigne, A. and Rozenberg, S. (2002) Epidemiology and prevention of ovarian hyperstimulation syndrome (OHSS): A review. Human Reproduction Update, 8, 559-577. doi:10.1093/humupd/8.6.559

[8] Aboulghar, M. (2009) Symposium: Update on prediction and management of OHSS. Prevention of OHSS. Reproductive Biomedicine Online, 19, 33-42. doi:10.1016/S1472-6483(10)60043-0

[9] Humaidan, P., Quartarolo, J. and Papanikolaou, E.G. (2010) Preventing ovarian hyperstimulation syndrome: Guidance for the clinician. Fertility and Sterility, 94, 389400. doi:10.1016/j.fertnstert.2010.03.028

[10] Delvigne, A. (2009) Symposium: Update on prediction and management of OHSS. Epidemiology of OHSS. Reproductive Biomedicine Online, 19, 8-13. doi:10.1016/S1472-6483(10)60040-5

[11] Papanikolaou, E.G., Humaidan, P., Polyzos, N.P. and Tarlatzis, B. (2010) Identification of the high-risk patient for ovarian hyperstimulation syndrome. Seminars in Reproductive Medicine, 28, 458-462. doi:10.1055/s-0030-1265671

[12] Delvigne, A., Demoulin, A., Smitz, J., Donnez, J., Koninckx, P., Dhont, M., Englert, Y., Delbeke, L., Darcis, L., Gordts, S., Puttemans, P., Gerris, J., Schoysman, R. and Leroy, F. (1993) The ovarian hyperstimulation syndrome in in-vitro fertilization: A Belgian multicentric study. I. Clinical and biological features. Human Reproduction, $\mathbf{8}$, 1353-1360.

[13] Enskog, A., Henriksson, M., Unander, M., Nilsson, L. and Brännström, M. (1999) Prospective study of the clinical and laboratory variables of patients in whom ovarian hyperstimulation syndrome developed during controlled ovarian hyperstimulation for in-vitro fertilization. Fertility and Sterility, 71, 808-814. doi:10.1016/S0015-0282(99)00090-4

[14] Tummon, I., Gavrilova-Jordan, L., Allemand, M.C. and Session, D. (2005) Polycystic ovaries and ovarian hyperstimulation syndrome: A systematic review. Acta Obstetrica et Gynecologica Scandinavica, 84, 611-616. doi:10.1080/j.0001-6349.2005.00788.x

[15] [Nakhuda, G.S., Chu, M.C., Wang, J.G., Sauer, M.W. and Lobo, R.A. (2006) Elevated serum müllerian-inhibiting substance may be a marker for ovarian hyperstimulation syndrome in normal women undergoing in-vitro fertilization. Fertility and Sterility, 85, 1541-1543. doi:10.1016/j.fertnstert.2005.10.052

[16] Practice Committee of the American Society of Reproductive Medicine (2008) Ovarian hyperstimulation syndrome. Fertility and Sterility, 90, S188-S193. doi:10.1016/j.fertnstert.2008.08.034

[17] Jayaprakasan, K., Jayaprakasan, R., Al-Hasie, H.A., Clewes, J.S., Campbell, B.K., Johnson, I.R. and RaineFenning, N.J. (2009) Can quantitative three-dimensional power Doppler angiography be used to predict ovarian hyperstimulation syndrome? Ultrasound in Obstetrics \& Gynecology, 33, 583-91. doi: $10.1002 /$ uog. 6373

[18] Luke, B., Brown, M.B., Morbeck, D.E., Hudson, S.B., Coddington 3rd, C.C. and Stern, J.E. (2010) Factors associated with ovarian hyperstimulation syndrome (OHSS) and its effect on assisted reproductive technology (ART) treatment and outcome. Fertility and Sterility, 94, 1399. 1404. doi:10.1016/j.fertnstert.2009.05.092

[19] Youssef, M.A., Van der Veen, F., Al-Inany, H.G., Griesinger, G., Mochtar, M.H., Aboulfoutouh, I., Khattab, S.M. and van Wely, M. (2011) Gonadotropin-releasing hormone agonist versus HCG for oocyte triggering in antagonist assisted reproductive technology cycles. Cochrane Database of Systematic Reviews, 1, Article ID: CD008046. doi:10.1002/14651858.CD008046.pub3

[20] Blankstein, J., Shalev, J., Saadon, T., Kukia, E.E., Rabinovici, J., Pariente, C., Lunenfeld, B., Serr, D.M. and Mashiach, S. (1987) Ovarian hyperstimulation syndrome: Prediction by number and size of preovulatory ovarian follicles. Fertility and Sterility, 47, 597-602.

[21] MacDougall, M.J., Tan, S.L. and Jacobs, H.S. (1992) Invitro fertilization and the ovarian hyperstimulation syndrome. Human Reproduction, 7, 597-600.

[22] Aboulghar, M. (2003) Prediction of ovarian hyperstimulation syndrome (OHSS). Estradiol level has an important role in the prediction of OHSS. Human Reproduction, 18, 1140-1141. doi:10.1093/humrep/deg208

[23] Asch, R.H., Li, H.P., Balmaceda, J.P., Weckstein, L.N. and Stone, S.C. (1991) Severe ovarian hyperstimulation 
syndrome in assisted reproductive technology: Definition of high risk groups. Human Reproduction, 6, 1395-1399.

[24] Mocanu, E., Redmond, M.L., Hennelly, B., Collins, C. and Harrison, R. (2007) Odds of ovarian hyperstimulation syndrome (OHSS)-Time for reassessment. Human Fertility (Cambridge), 10, 175-181. doi:10.1080/14647270701194143

[25] Aramwit, P., Pruksananonda, K., Kasettratat, N. and Jammeechai, K. (2008) Risk factors for ovarian hyperstimulation syndrome in Thai patients using gonadotropins for in vitro fertilization. American Journal of Health-System Pharmacy, 65, 1148-1153. doi:10.2146/ajhp070566

[26] Álvarez, C., Martí-Bonmatí, L., Novella-Maestre, E., Sanz, R., Gómez, R., Fernández-Sánchez, M., Simón, C. and Pellicer, A. (2007) Dopamine agonist cabergoline reduces hemoconcentration and ascites in hyperstimulated women undergoing assisted reproduction. Journal of Clinical Endocrinology \& Metabolism, 92, 2931-2937. doi:10.1210/jc.2007-0409

[27] Busso, C.E., Garcia-Velasco, J., Gomez, R., Alvarez, C., Simón, C. and Pellicer, A. (2009) Symposium: Update on prediction and management of OHSS. Prevention of OHSS-Dopamine agonists. Reproductive Biomedicine Online, 19, 43-51. doi:10.1016/S1472-6483(10)60044-2

[28] D'Angelo, A., Brown, J. and Amso, N.N. (2011) Coasting (withholding gonadotrophins) for preventing ovarian hyperstimulation syndrome. Cochrane Database of Systematic Reviews, 6, Article ID: CD002811. doi:10.1002/14651858.CD002811.pub2

[29] Tang, H., Hunter, T., Hu, Y., Zhai, S.D., Sheng, X. and Hart, R.J. (2012) Cabergoline for preventing ovarian hyperstimulation syndrome. Cochrane Database of Systematic Reviews, 2, Article ID: CD008605. doi:10.1002/14651858.CD008605.pub2

[30] Busso, C., Fernández-Sánchez, M., García-Velasco, J.A., Landeras, J., Ballesteros, A., Muñoz, E., González, S., Simón, C., Arce, J.C. and Pellicer, A. (2010) The nonergot derived dopamine agonist quinagolide in prevention of early ovarian hyperstimulation syndrome in IVF patients: A randomized, double-blind, placebo-controlled trial. Human Reproduction, 25, 95-1004. doi:10.1093/humrep/deq005

[31] Donoho, D. and Jin, J. (2008) Higher criticism thresholding: Optimal feature selection when useful features are rare and weak. Proceedings of the National Academy of Sciences, 105, 14790-14795. doi:10.1073/pnas.0807471105

[32] Danninger, B., Brunner, M., Obruca, A. and Feichtinger, W. (1996) Prediction of ovarian hyperstimulation syndrome by ultrasound volumetric assessment [corrected] of baseline ovarian volume prior to stimulation. Human Reprodution, 11, 1597-1599. doi:10.1093/oxfordjournals.humrep.a019451
[33] Navot, D., Bergh, P.A. and Laufer, N. (1992) Ovarian hyperstimulation syndrome in novel reproductive technologies: Prevention and treatment. Fertility and Sterility, 58, 249-261.

[34] Jayaprakasan, K., Herbert, M., Moody, E., Stewart, J.A. and Murdoch, A.P. (2007) Estimating the risks of ovarian hyperstimulation syndrome (OHSS): Implications for egg donation for research. Human Fertility (Cambridge), 10, 183-187. doi:10.1080/14647270601021743

[35] Dahl Lyons, C.A., Wheeler, C.A., Frishman, G.N., Hackett, R.J., Seifer, D.B. and Haning, R.V. (1994) Early and late presentations of the ovarian hyperstimulation syndrome: Two distinct entities with different risk factors. Human Reproduction, 9, 792-799.

[36] Oyesanya, O.A., Parsons, J.H., Collins, W.P. and Campbell, S. (1995) Total ovarian volume before human chorionic gonadotrophin administration for ovulation induction may predict the hyperstimulation syndrome. Human Reproduction, 10, 3211-3212.

[37] Haning Jr., R.V., Austin, C.W., Carlson, I.H., Kuzma, D.L., Shapiro, S.S. and Zweibel, W.J. (1983) Plasma estradiol is superior to ultrasound and urinary estriol glucuronide as a predictor of ovarian hyperstimulation during induction of ovulation with menotropins. Fertility and Sterility, 40, 31-36.

[38] Hendriks, D.J., Klinkert, E.R., Bancsi, L.F., Looman, C.W., Habbema, J.D., te Velde, E.R. and Broekmans, F.J. (2004) Use of stimulated serum estradiol measurements for the prediction of hyperresponse to ovarian stimulation in in vitro fertilization (IVF). Journal of Assisted Reproduction and Genetics, 21, 65-72. doi:10.1023/B:JARG.0000027016.65749.ad

[39] Papanikolaou, E.G., Pozzobon, C., Kolibianakis, E.M., Camus, M., Tournaye, H., Fatemi, H.M., Van Steirteghem, A. and Devroey, P. (2006) Incidence and prediction of ovarian hyperstimulation syndrome in women undergoing gonadotropin-releasing hormone antagonist in vitro fertilization cycles. Fertility and Sterility, 85, 112-120. doi:10.1016/j.fertnstert.2005.07.1292

[40] Stolwijk, A.M., Zielhuis, G.A., Hamilton, C.J., Straatman, H., Hollanders, J.M., Goverde, H.J., van Dop, P.A. and Verbeek, A.L. (1996) Prognostic models for the probability of achieving an ongoing pregnancy after in-vitro fertilization and the importance of testing their predictive value. Human Reproduction, 11, 2298-2303. doi:10.1093/oxfordjournals.humrep.a019092

[41] Stolwijk, A.M., Straatman, H., Zielhuis, G.A., Jansen, C.A., Braat, D.D., van Dop, P.A. and Verbeek, A.L. (1998) External validation of prognostic models for ongoing pregnancy after in-vitro fertilization. Human Reproduction, 13, 3542-3549. doi:10.1093/humrep/13.12.3542. 